\title{
Arginine-containing desensitizing toothpaste for the treatment of dentin hypersensitivity: a meta-analysis
}

\author{
Zheng-yan Yang ${ }^{1,2}$ \\ Fei Wangl,2 \\ Keke Lu ${ }^{1,2}$ \\ Yue-heng $\mathrm{Li}^{1,2}$ \\ Zhi Zhou',2 \\ 'Department of Preventive Dentistry, \\ The College of Stomatology, \\ ${ }^{2}$ Department of Preventive Dentistry, \\ The Affiliated Hospital of Stomatology, \\ Chongqing Medical University, \\ Chongqing, People's Republic of China
}

Correspondence: Zhi Zhou

The Affiliated Hospital of Stomatology, Chongqing Medical University, No 426, Songshibei Road, Yubei District,

Chongqing 40II47, People's Republic of China

Tel +86 I30 6238 |906

Fax +862388860222

Email zhouzhi_050918@hotmail.com
This article was published in the following Dove Press journal:

Clinical, Cosmetic and Investigational Dentistry

7 January 2016

Number of times this article has been viewed

Objective: To estimate the effect of arginine-containing desensitizing toothpaste on dentin hypersensitivity (DH).

Methods: Databases including China National Knowledge Infrastructure, VIP Database for Chinese Technical Periodicals, China Biology Medicine disc, Wangfang Data, PubMed, Web of Science, and Cochrane Trials Register were searched, and Google was used as a supplementary tool to search for information through February 2014. Randomized controlled trials (RCTs) of the treatment of DH with arginine-containing toothpaste were included. Relevant information was extracted, and a quality evaluation was performed. Meta-analyses were performed using RevMan 5.2 software.

Results: Eighteen RCTs with 1,423 patients were included. The results of the meta-analyses demonstrated that at days 0 and 3; weeks 2, 4, and 8; and more than 12 weeks, argininecontaining toothpaste led to significantly improved results on the tactile sensitivity test (standardized mean difference $[\mathrm{SMD}]=1.95,95 \%$ confidence interval $[\mathrm{CI}][1.14,2.76]$ ) and the air-blast test (SMD $=-1.60,95 \% \mathrm{CI}[-2.14,-1.05])$ at 4 weeks and the tactile sensitivity test $(\mathrm{SMD}=2.01,95 \% \mathrm{CI}[1.41,2.61])$ and the air-blast test $(\mathrm{SMD}=-1.41,95 \% \mathrm{CI}[-1.83,-0.98])$ at 8 weeks compared to toothpastes containing other desensitizing components, thus indicating a superior therapeutic effect of arginine-containing desensitizing toothpaste. However, no significant differences between arginine-containing toothpaste and toothpastes containing other desensitizing components were observed in the air-blast test at days 0 and 3 and week 2 and in the tactile sensitivity and air-blast tests at more than 12 weeks.

Conclusion: The current evidence indicates that arginine-containing toothpaste is effective for DH. However, further high-quality, large-sample RCTs are needed.

Keywords: arginine-containing toothpastes, dentin hypersensitivity, meta-analysis, randomized controlled trial

\section{Introduction}

Dentin hypersensitivity (DH) is a frequently reported dental condition that is typically characterized by brief, sharp pain that cannot be ascribed to any other form of dental defect or pathology and arises from exposed dentin in response to thermal, evaporative, tactile, osmotic, or chemical stimuli. ${ }^{1} \mathrm{DH}$ is increasingly recognized as an important issue to be addressed from both diagnostic and problem-management perspectives because the improved success of caries prevention and periodontal disease management measures have resulted in improved oral health status and dentition function throughout life. The reported prevalence of $\mathrm{DH}$ ranges from $2 \%$ to $57 \%$ worldwide. ${ }^{2}$ A commonly supported mechanism for DH is the hydrodynamic theory, 
which assumes that painful stimulation increases fluid flow within the dentinal tubules, causing activation of resident baroceptors. ${ }^{3}$ Accordingly, the ideal treatment for DH should reduce fluid flow within the dentinal tubules or block the pulp nerve response. Dozens of in-office sensitivity treatments and mass-market sensitivity relief toothpastes are available worldwide, and fluoride dentifrice is generally accepted. There are two types of sensitivity relief toothpastes. One type contains potassium salts; the potassium ions have a depolarizing effect on electrical nerve conduction, thereby causing nerve fibers to be less excitable by stimuli ${ }^{4}$ and, consequently, reducing the patient's sensation of pain. The other type of sensitivity relief toothpaste includes dentifrices that occlude the exposed dentin tubules to block the hydrodynamic mechanism of pain stimulation. Examples include strontium-containing toothpaste and stannous-containing toothpaste, ${ }^{5-10}$ but the effects of these toothpastes are inferior to potassium-containing toothpastes. Clinical studies have demonstrated the efficacy of arginine-containing dentifrices in reducing DH. ${ }^{11-29}$ To assess whether arginine-containing dentifrices are efficacious in reducing DH compared with toothpastes containing potassium, strontium, or fluoride, a systematic review of the literature is required. We therefore performed a meta-analysis to assess the efficacy of arginine-containing dentifrices compared with toothpastes containing potassium, strontium, or fluoride for future clinical applications.

\section{Materials and methods}

All experimental procedures in this study were approved by the ethics committee of the Stomatology Hospital Affiliated to Chongqing Medical University.

\section{Eligibility criteria}

1. Inclusion criteria.

1.1. Research design: randomized controlled trials (RCTs) published in full; there were no limitations on language.

1.2. Patient: studies performed on adult humans (age $>18$ years) with a clinical diagnosis of $\mathrm{DH}$ caused by cervical dentin exposure.

1.3. Intervention and comparison: the test group used an arginine-containing dentifrice daily, whereas the control group used toothpaste containing other desensitizing components daily.

1.4. Outcome: the results were evaluated based on the score of the improvement of dentin sensitivity (tactile sensitivity and air-blast sensitivity).
2. Exclusion criteria: 1) in vitro study; 2) review literature; 3) studies not reporting complete data; and 4) repeated published literature or papers with duplicate data.

\section{Literature search of clinical trials}

A thorough literature search of computerized databases, including PubMed, Cochrane Controlled Clinical Trial Register (First Journal, 2014), Web of Science, China National Knowledge Infrastructure, VIP Database for Chinese Technical Periodicals, China Biology Medicine disc, and the Wangfang Data, was performed through February 2014 by two independent researchers to identify RCTs of arginine-containing dentifrices compared with toothpastes containing other desensitizing components with a curative effect on DH. To avoid selection bias, no restrictions were applied with regard to language or year. Moreover, to identify unpublished literature and further examine trends in the literature, the Google search engine was used.

The Chinese keywords were arginine (精氨酸), toothpaste (牙膏), tooth (牙齿), dentin (牙本质), and hypersensitivity (过敏,敏感). The English keywords were toothpaste, tooth paste, dentifrice, dentifrices, desensitize, agent, efficacy, effect, dentin, dentine, tooth, teeth, root, hypersensitivity, hypersensitive, sensitivity, sensitive, and oversensitive. In the case of PubMed, the specific retrieval strategy presented in Figure 1 was used.

\section{Literature screening, data extraction, and quality assessment}

Literature reviews were performed by two independent evaluators to include and exclude literature according to the literature and to cross-check the details. The title was first read; if the title met the eligibility criteria, the full text was read. If the full text met the eligibility criteria, the study was included. Disagreements were resolved in consultation with a third researcher.

The data was then extracted to a table and the RCT bias risk assessment tool recommended by the Cochrane Reviewers' Handbook 5.1.0 $0^{30}$ was used to evaluate the risk of bias in the included studies.

\section{Statistical analysis}

Data were combined for the meta-analysis using a statistical software package (RevMan software, version 5.2, The Nordic Cochrane Center, The Cochrane Collaboration, Copenhagen, Denmark). Continuous outcomes used the standardized mean difference (SMD) or mean difference (MD) with 95\% confidence intervals (CIs) as effect measures, whereas 


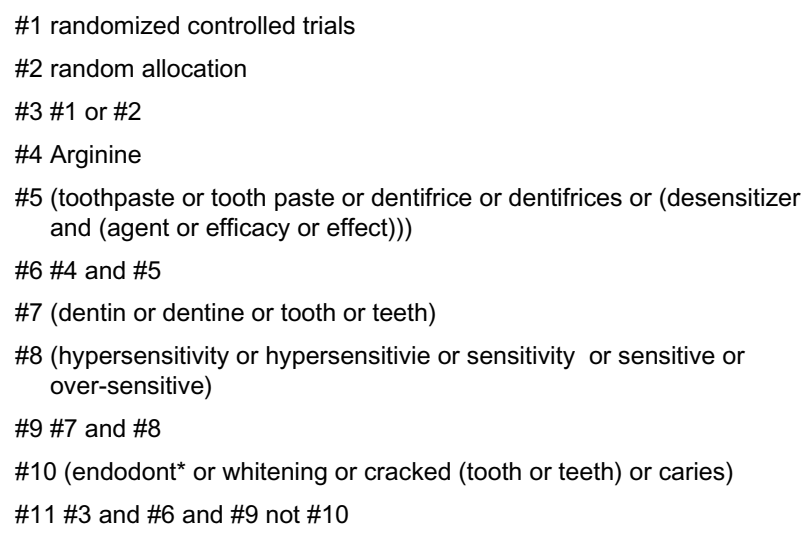

Figure I Specific retrieval strategy for PubMed searches.

Note: *Wildcard symbol for truncation searching.

dichotomous outcomes used the risk ratio with $95 \%$ CIs as effect measures. Heterogeneity was assessed using the $\chi^{2}$-based method and $I^{2}$ measurement. $P$-values and $95 \%$ CIs were then calculated. For $P<0.1$ and $I^{2}>50 \%$, the random-effect model was used; otherwise, the fixed-effect model was used. Descriptive analysis was performed when the data could not be combined.

\section{Sensitivity analysis}

Sensitivity analysis was performed by removing one result and performing statistical analysis again. The new result was compared with the original to explore the effects of removing the result on the effect size. If no difference was observed, the results of the meta-analysis were considered reliable.

\section{Grading of the evidence}

The GRADE (Grading of Recommendations Assessment, Development, and Evaluation) approach was adopted to evaluate the overall quality of the evidence. ${ }^{31,32}$ We applied the following definitions of quality of the evidence. ${ }^{33}$

- High quality: further research is unlikely to change confidence in the estimate of the effect. There are no known or suspected reporting biases; all domains are fulfilled.

- Moderate quality: further research is likely to have an important effect on confidence in the estimate of the effect and might change the estimate; one of the domains was not fulfilled.

- Low quality: further research is likely to have an important effect on confidence in the estimate of the effect and is likely to change the estimate; two of the domains were not fulfilled.

- Very low quality: we are uncertain about the estimate; three of the domains were not fulfilled.

\section{Results}

\section{Literature search}

Electronic and manual searches identified 382 articles. After step-by-step screening, 18 studies including 1,423 patients were qualified for inclusion in the meta-analysis. A PRISMA (Preferred Reporting Items for Systematic Reviews and MetaAnalyses) flowchart of the complete study-selection process is illustrated in Figure 2. The basic characteristics of the included studies are presented in Table 1. The methodological quality analysis of the included studies is presented in Table 2.

\section{Meta-analysis results}

Based on the interventions evaluated, the included studies were classified into three types: arginine-containing dentifrice compared with potassium-containing toothpastes, arginine-containing dentifrice compared with strontium-containing toothpastes,

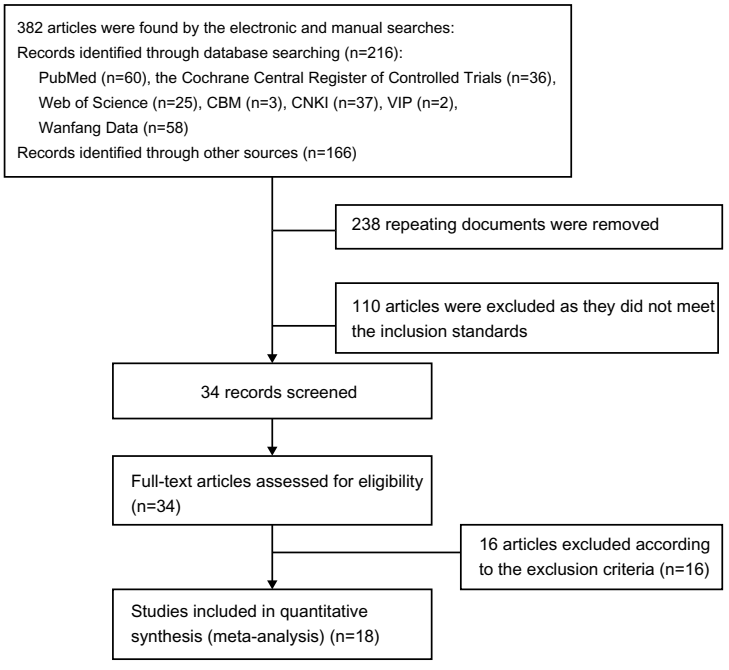

Figure 2 PRISMA flowchart of the search strategy.

Abbreviations: CBM, China Biology Medicine; CNKI, China National Knowledge Infrastructure; PRISMA, Preferred Reporting Items for Systematic Reviews and MetaAnalyses. 


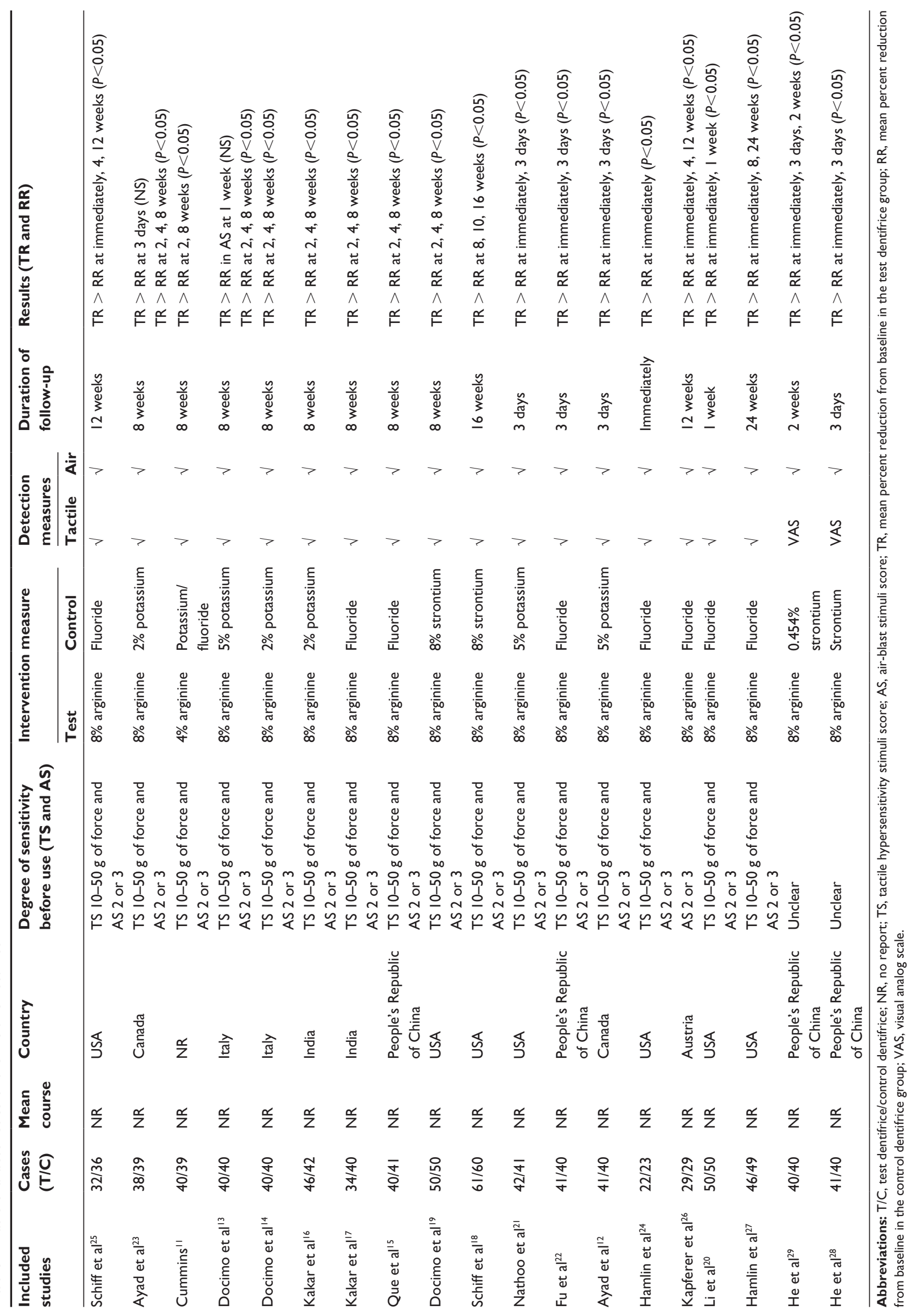


Table 2 Methodological quality analysis of the included studies

\begin{tabular}{|c|c|c|c|c|c|c|}
\hline $\begin{array}{l}\text { Included } \\
\text { studies }\end{array}$ & $\begin{array}{l}\text { Sequence } \\
\text { generation }\end{array}$ & $\begin{array}{l}\text { Allocation } \\
\text { concealment }\end{array}$ & $\begin{array}{l}\text { Blinding } \\
\text { participants }\end{array}$ & $\begin{array}{l}\text { Free of incomplete } \\
\text { data bias }\end{array}$ & $\begin{array}{l}\text { Free of selective } \\
\text { reporting }\end{array}$ & $\begin{array}{l}\text { Other sources } \\
\text { of bias }\end{array}$ \\
\hline Schiff et a ${ }^{25}$ & Unclear & Unclear & Yes & Yes & No & No \\
\hline Ayad et $\mathrm{al}^{23}$ & Unclear & Yes & Yes & Yes & No & No \\
\hline Cummins" & Unclear & Unclear & Yes & Yes & No & No \\
\hline Docimo et $\mathrm{al}^{13}$ & Unclear & Yes & Yes & Yes & No & No \\
\hline Docimo et $\mathrm{al}^{14}$ & Unclear & Yes & Yes & Yes & No & No \\
\hline Kakar et $\mathrm{al}^{16}$ & Unclear & Yes & Yes & Yes & No & No \\
\hline Kakar et a $\left.\right|^{17}$ & Unclear & Yes & Yes & Yes & No & No \\
\hline Que et al ${ }^{15}$ & Yes & Unclear & Yes & Yes & No & No \\
\hline Docimo et $\mathrm{al}^{19}$ & Unclear & Unclear & Yes & Yes & No & No \\
\hline Schiff et al ${ }^{18}$ & Yes & Yes & Yes & Yes & No & No \\
\hline Nathoo et $\mathrm{al}^{21}$ & Unclear & Unclear & Yes & Yes & No & No \\
\hline Fu et $\mathrm{al}^{22}$ & Unclear & Unclear & Yes & Yes & No & No \\
\hline Ayad et al ${ }^{12}$ & Unclear & Yes & Yes & Yes & No & No \\
\hline Hamlin et $\mathrm{a}^{24}$ & Unclear & Unclear & Yes & Yes & No & No \\
\hline Kapferer et $\mathrm{al}^{26}$ & Unclear & Unclear & Yes & Yes & No & No \\
\hline Li et $\mathrm{al}^{20}$ & Unclear & Yes & Yes & Yes & No & No \\
\hline Hamlin et $\mathrm{al}^{27}$ & Unclear & Unclear & Yes & Yes & No & No \\
\hline $\mathrm{He}$ et $\mathrm{al}^{28}$ & Unclear & Unclear & Yes & Yes & No & No \\
\hline $\mathrm{He}$ et $\mathrm{a}^{29}$ & Unclear & Unclear & Yes & Yes & No & No \\
\hline
\end{tabular}

and arginine-containing dentifrice compared with fluoride toothpastes. We evaluated the tactile and air-blast DH scores of the arginine-containing dentifrice with control toothpastes at days 0 and 3; weeks 2,4 , and 8 ; and more than 12 weeks.

\section{Comparison of instant tactile hypersensitivity between arginine- containing dentifrice group and the control group}

Instant tactile hypersensitivity was compared between the arginine-containing dentifrice group (313 persons) and the control group (321 persons). A random-effect model was used in the meta-analysis due to statistical heterogeneity among the studies. The analysis excluded Kapferer et al's study ${ }^{26}$ (because the 95\% CI lines were invalid vertical lines and the results were not significant). The mean tactile hypersensitivity scores measured immediately ( 0 day) were significantly higher for the arginine-containing dentifrice group than the control group (SMD $=1.66,95 \%$ CI $[1.00,2.31], P<0.00001)$, indicating that the arginine-containing dentifrice was superior to toothpastes containing other desensitizing components (Figure 3).

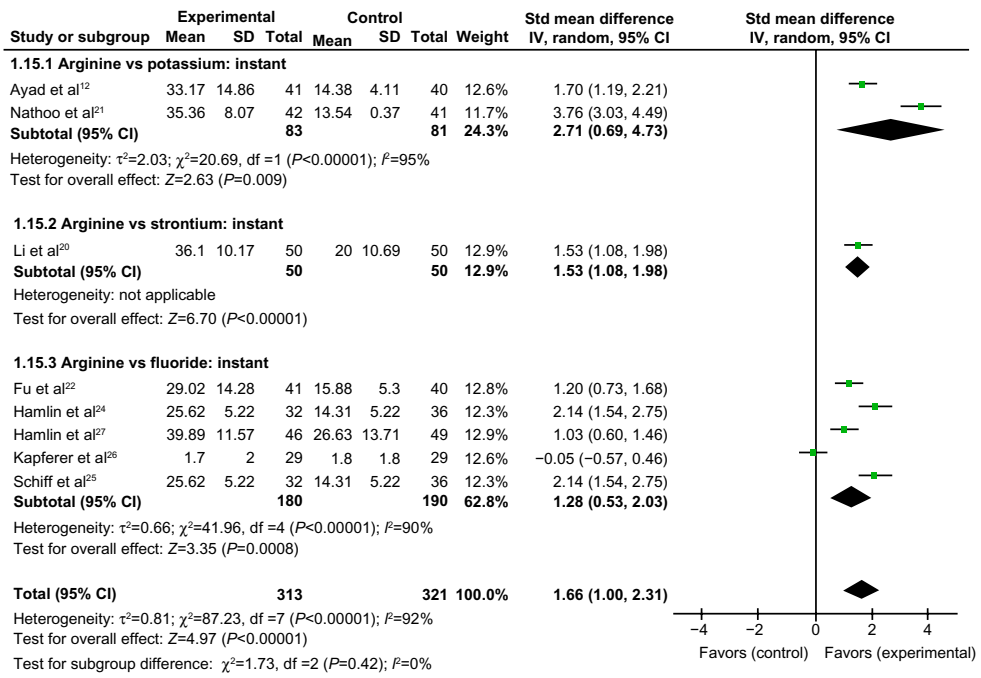

Figure 3 Meta-analysis of the comparison of instant tactile hypersensitivity between the arginine-containing dentifrice group and the control group. Abbreviations: SD, standard deviation; $\mathrm{Cl}$, confidence interval; Std, standardized; df, degree of freedom. 


\section{Comparison of instant air-blast hypersensitivity between the arginine- containing dentifrice group and control group}

Instant air-blast hypersensitivity was compared between the arginine-containing dentifrice group (384 persons) and the control group (388 persons). A random-effect model was used in the meta-analysis due to statistical heterogeneity among the studies. Because the total 95\% CI lines were invalid vertical lines and the results were not significant, we concluded that the immediate effects of the arginine-containing dentifrice and toothpastes containing other desensitizing components for the treatment of $\mathrm{DH}$ did not differ significantly (Figure 4).

\section{Comparison of 3-day tactile hypersensitivity between the arginine- containing dentifrice group and control group}

Three-day tactile hypersensitivity was compared between the arginine-containing dentifrice group (162 persons) and the control group (160 persons). A random-effect model was used in the meta-analysis due to statistical heterogeneity among the studies. The mean tactile hypersensitivity scores were significantly higher in the arginine-containing dentifrice group than the control group at 3 days (SMD $=2.21,95 \%$ CI [1.13, 3.29], $P<0.0001)$. We thus concluded that the arginine-containing dentifrice was superior to toothpastes containing other desensitizing components (Figure 5).

\section{Comparison of 3-day air-blast hypersensitivity between the arginine- containing dentifrice group and control group}

Three-day air-blast hypersensitivity was compared between the arginine-containing dentifrice group (202 persons) and the control group (200 persons). A random-effect model was used in the meta-analysis due to statistical heterogeneity among the studies. Ayad et al's study ${ }^{23}$ was excluded because the total 95\% CI lines were invalid vertical lines and the results were not significant. We concluded that the effects of the argininecontaining dentifrice and the toothpastes containing other desensitizing components did not differ significantly for the treatment of $\mathrm{DH}$ at 3 days (Figure 6).

\section{Comparison of 2-week tactile hypersensitivity between the arginine- containing dentifrice group and control group}

Two-week tactile hypersensitivity was compared between the arginine-containing dentifrice group (323 persons) and the control group (327 persons). A random-effect model was used in the meta-analysis due to statistical heterogeneity among the studies. The mean tactile hypersensitivity scores were significantly higher in the arginine-containing dentifrice group than the control group at 2 weeks $(\mathrm{SMD}=1.20,95 \%$ CI $[0.84,1.57], P<0.00001)$. We thus concluded that the arginine-containing dentifrice was superior to toothpastes containing other desensitizing components (Figure 7).

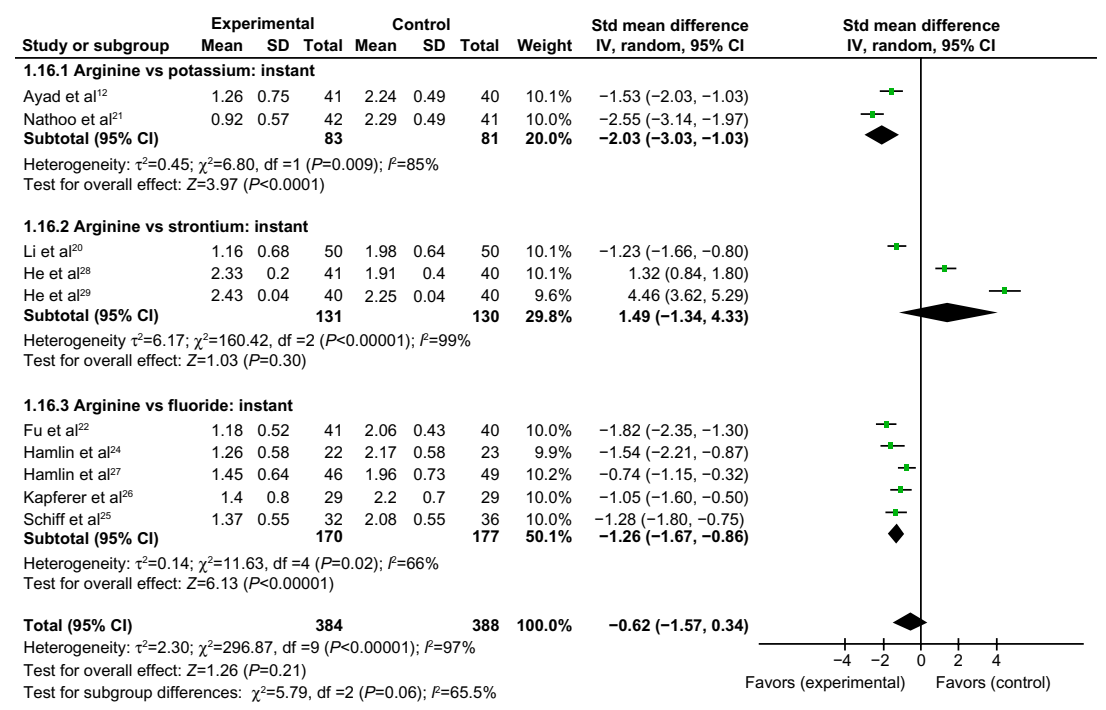

Figure 4 Meta-analysis of the comparison of instant air-blast hypersensitivity between the arginine-containing dentifrice group and control group. Abbreviations: SD, standard deviation; $\mathrm{Cl}$, confidence interval; Std, standardized; $d f$, degree of freedom. 


\begin{tabular}{|c|c|c|c|c|c|c|c|c|c|c|}
\hline \multirow[t]{2}{*}{ Study or subgroup } & \multicolumn{3}{|c|}{$\begin{array}{l}\text { Experimental } \\
\text { Men }\end{array}$} & \multicolumn{3}{|c|}{ Control } & Weight & \multirow[t]{2}{*}{$\begin{array}{l}\text { Std mean difference } \\
\text { IV, random, } 95 \% \mathrm{CI}\end{array}$} & \multicolumn{2}{|c|}{$\begin{array}{l}\text { Std mean difference } \\
\mathrm{IV} \text {, random, } 95 \% \mathrm{Cl}\end{array}$} \\
\hline & \multicolumn{9}{|c|}{ 1.17.1 Arginine vs potassium: 3 -day } & \\
\hline Ayad et $\mathrm{a}^{\mathrm{P}^{33}}$ & 13.77 & 0 & 38 & 13.77 & 0 & 39 & & Not estimable & & \\
\hline Ayad et al|12 & 33.29 & 14.69 & 41 & 16.25 & 4.77 & 40 & $34.1 \%$ & $1.54(1.04,2.04)$ & & - \\
\hline Nathoo et a $\left.\right|^{21}$ & 39.17 & 7.72 & 42 & 15.58 & 5.47 & 41 & $31.9 \%$ & $3.49(2.79,4.18)$ & & \\
\hline Subtotal $(95 \% \mathrm{Cl})$ & & & 121 & & & 120 & $66.0 \%$ & $2.50(0.59,4.41)$ & & \\
\hline \multicolumn{11}{|c|}{ Heterogeneity: $\tau^{2}=1.80 ; \chi^{2}=20.01, \mathrm{df}=1(P<0.00001) ; R=95 \%$} \\
\hline \multicolumn{11}{|c|}{ Test for overall effect: $Z=2.56(P=0.01)$} \\
\hline \multicolumn{11}{|c|}{ 1.17.2 Arginine vs fluoride: 3 -day } \\
\hline Fu et a $\left.\right|^{22}$ & 33.41 & 13.06 & 41 & 16 & 5.91 & 40 & $34.0 \%$ & $1.69(1.18,2.21)$ & & - \\
\hline Subtotal $(95 \% \mathrm{Cl})$ & & & 41 & & & 40 & $34.0 \%$ & $1.69(1.18,2.21)$ & & $\bullet$ \\
\hline \multicolumn{11}{|c|}{ Heterogeneity: not applicable } \\
\hline \multicolumn{11}{|c|}{ Test for overall effect: $Z 6.50(P<0.00001)$} \\
\hline Total $(95 \% \mathrm{Cl})$ & & & 162 & & & 160 & $100.0 \%$ & $2.21(1.13,3.29)$ & & \\
\hline \multicolumn{9}{|c|}{$\begin{array}{l}\text { Heterogeneity: } \tau^{2}=0.83 ; \chi^{2}=22.34, \text { df }=2(P<0.0001) ; P=91 \% \\
\text { Test for overall effect: } Z 4.01(P<0.0001)\end{array}$} & $\begin{array}{ccc}1 & 1 & 1 \\
-4 & -2 & 0\end{array}$ & 2 \\
\hline \multicolumn{9}{|c|}{ Test for subgroup differences: $\chi^{2}=0.63, \mathrm{df}=1(P=0.43) ; R=0 \%$} & & \\
\hline
\end{tabular}

Figure 5 Meta-analysis of the comparison of 3-day tactile hypersensitivity between the arginine-containing dentifrice group and control group. Abbreviations: SD, standard deviation; Cl, confidence interval; Std, standardized; df, degree of freedom.

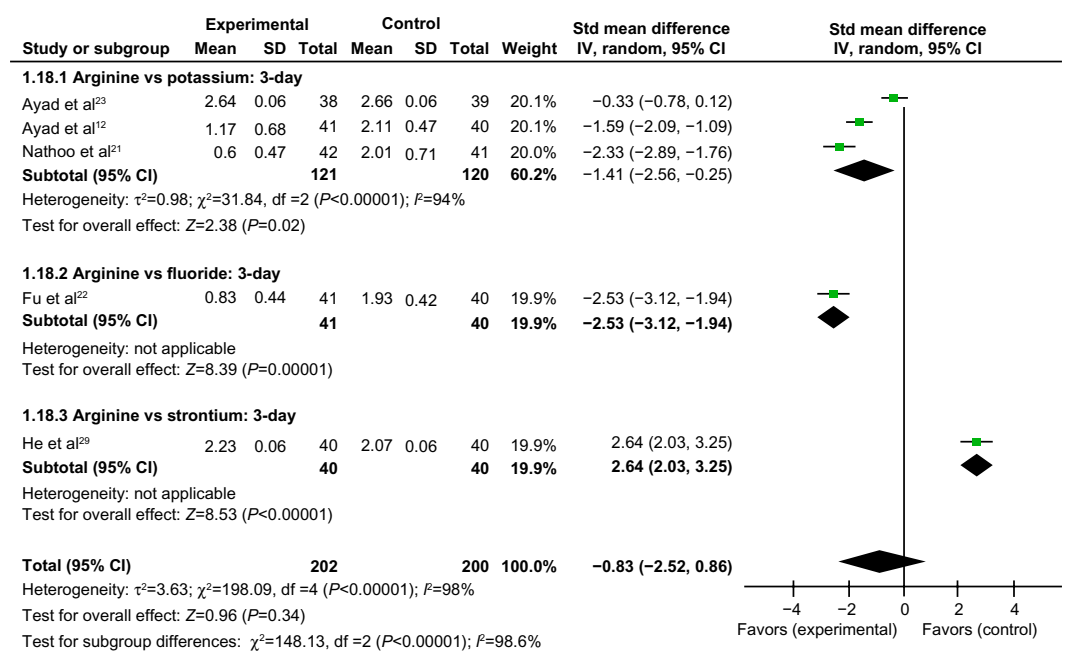

Figure 6 Meta-analysis of the comparison of 3-day air-blast hypersensitivity between the arginine-containing dentifrice group and control group. Abbreviations: SD, standard deviation; $\mathrm{Cl}$, confidence interval; Std, standardized; df, degree of freedom.

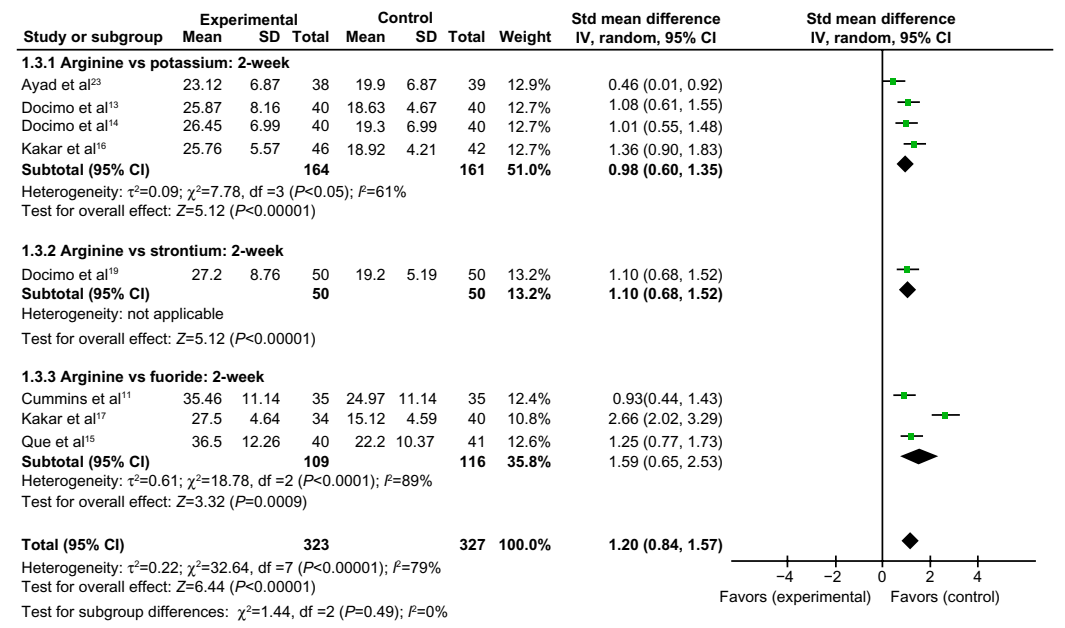

Figure 7 Meta-analysis of the comparison of 2-week tactile hypersensitivity between the arginine-containing dentifrice group and control group. Abbreviations: SD, standard deviation; $\mathrm{Cl}$, confidence interval; Std, standardized; df, degree of freedom. 


\section{Comparison of 2-week air-blast} hypersensitivity between the argininecontaining dentifrice group and the control group

Two-week air-blast hypersensitivity was compared between the arginine-containing dentifrice group (404 persons) and the control group (407 persons). A random-effect model was used in the meta-analysis due to statistical heterogeneity among the studies. Because the total 95\% CI lines were invalid vertical lines and the results were not significant, we concluded that the effects of the arginine-containing dentifrice and toothpastes containing other desensitizing components did not differ significantly for the treatment of $\mathrm{DH}$ at 2 weeks (Figure 8).

\section{Comparison of 4-week tactile hypersensitivity between the arginine- containing dentifrice group and control group}

Four-week tactile hypersensitivity was compared between the arginine-containing dentifrice group (349 persons) and the control group (357 persons). A random-effect model was used in the meta-analysis due to statistical heterogeneity among the studies. Kapferer et al's study ${ }^{26}$ was excluded because the $95 \%$ CI lines were invalid vertical lines and the results were not significant. The mean tactile hypersensitivity scores were significantly higher in the arginine-containing dentifrice group than the control group at 4 weeks (SMD $=1.95,95 \% \mathrm{CI}[1.14,2.76], P<0.00001)$. We therefore concluded that the arginine-containing dentifrice was superior to toothpastes containing other desensitizing components (Figure 9).

\section{Comparison of 4-week air-blast hypersensitivity between the arginine- containing dentifrice group and control group}

Four-week air-blast hypersensitivity was compared between the arginine-containing dentifrice group (349 persons) and the control group (357 persons). A random-effect model was used in the meta-analysis due to statistical heterogeneity among the studies. Docimo et al's study ${ }^{13}$ was excluded because the 95\% CI lines were invalid vertical lines and the results were not significant. The mean air-blast hypersensitivity scores were significantly higher in the arginine-containing dentifrice group than the control group at 4 weeks $(\mathrm{SMD}=-1.60,95 \%$ CI $[-2.14,-1.05], P<0.00001)$. Thus, we concluded that the arginine-containing dentifrice was superior to toothpastes containing other desensitizing components (Figure 10).

\section{Comparison of 8-week tactile hypersensitivity between the arginine- containing dentifrice group and control group}

Eight-week tactile hypersensitivity was compared between the arginine-containing dentifrice group (435 persons) and the control group (440 persons). A random-effect model was used in the meta-analysis due to statistical heterogeneity among the studies. The mean tactile hypersensitivity scores were significantly higher in the arginine-containing dentifrice

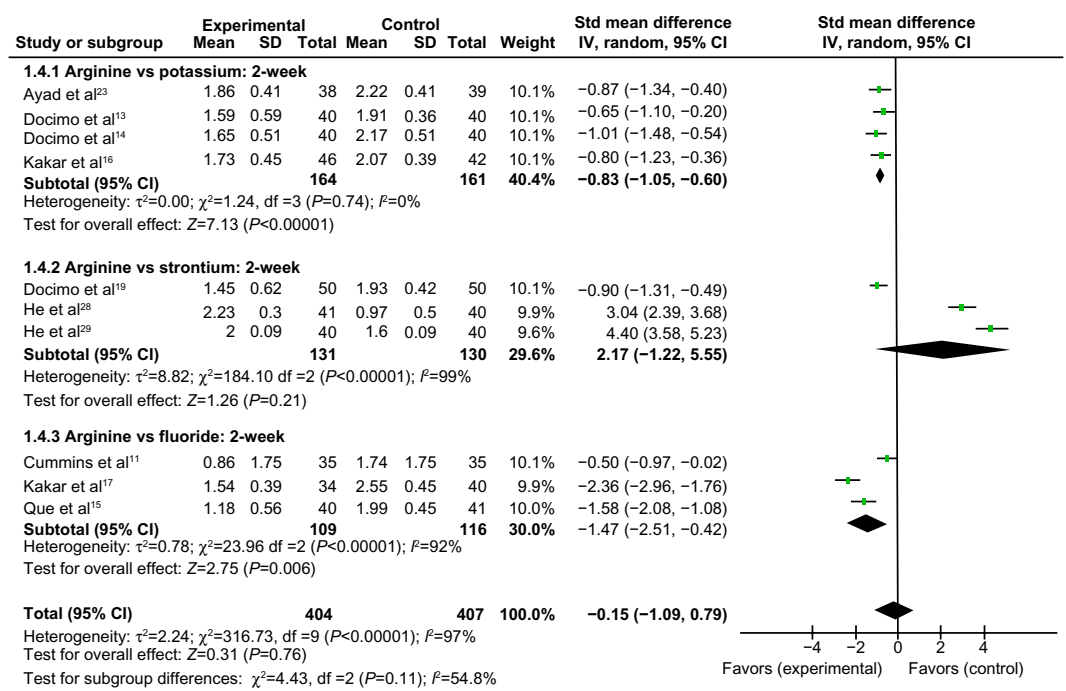

Figure 8 Meta-analysis of the comparison of 2-week air-blast hypersensitivity between the arginine-containing dentifrice group and control group. Abbreviations: SD, standard deviation; $\mathrm{Cl}$, confidence interval; Std, standardized; df, degree of freedom. 


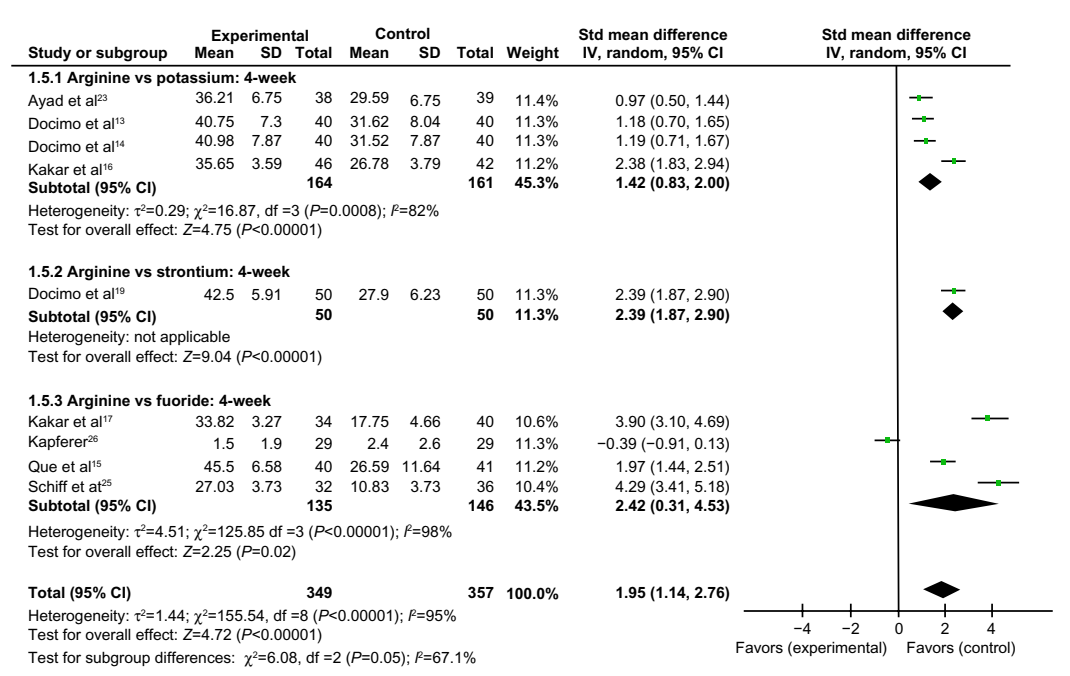

Figure 9 Meta-analysis of the comparison of 4-week tactile hypersensitivity between the arginine-containing dentifrice group and control group. Abbreviations: SD, standard deviation; $\mathrm{Cl}$, confidence interval; Std, standardized; $\mathrm{df}$, degree of freedom.

group than the control group at 8 weeks (SMD $=2.01,95 \%$ CI $[1.41,2.61], P<0.00001)$. Thus, we concluded that the arginine-containing dentifrice was superior to toothpastes containing other desensitizing components (Figure 11).

\section{Comparison of 8-week air-blast hypersensitivity between the arginine- containing dentifrice group and control group}

Eight-week air-blast hypersensitivity was compared between the arginine-containing dentifrice group (435 persons) and the control group (440 persons). A random-effect model was used in the meta-analysis due to statistical heterogeneity among the studies. Cummins' study ${ }^{11}$ and Docimo et al's study ${ }^{14}$ were excluded because the $95 \%$ CI lines were invalid vertical lines and the results were not significant. The mean air-blast hypersensitivity scores were significantly higher in the arginine-containing dentifrice group than the control group at 8 weeks ( $\mathrm{SMD}=-1.41$, $95 \%$ CI $[-1.83,-0.98], P<0.00001)$. Thus, we concluded that the arginine-containing dentifrice was superior to toothpastes containing other desensitizing components (Figure 12).

\section{Comparison of $>12$-week tactile hypersensitivity and air-blast hypersensitivity between the arginine-containing dentifrice group and control group}

Tactile hypersensitivity and air-blast hypersensitivity at time points longer than 12 weeks were compared between the

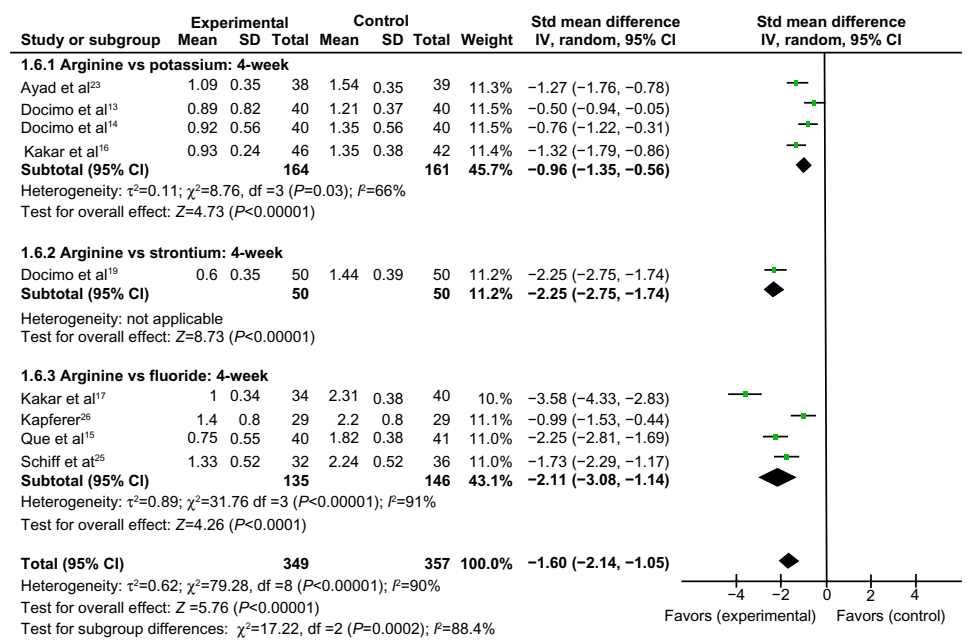

Figure 10 Meta-analysis of the comparison of 4-week air-blast hypersensitivity between the arginine-containing dentifrice group and control group. Abbreviations: SD, standard deviation; $\mathrm{Cl}$, confidence interval; Std, standardized; df, degree of freedom. 


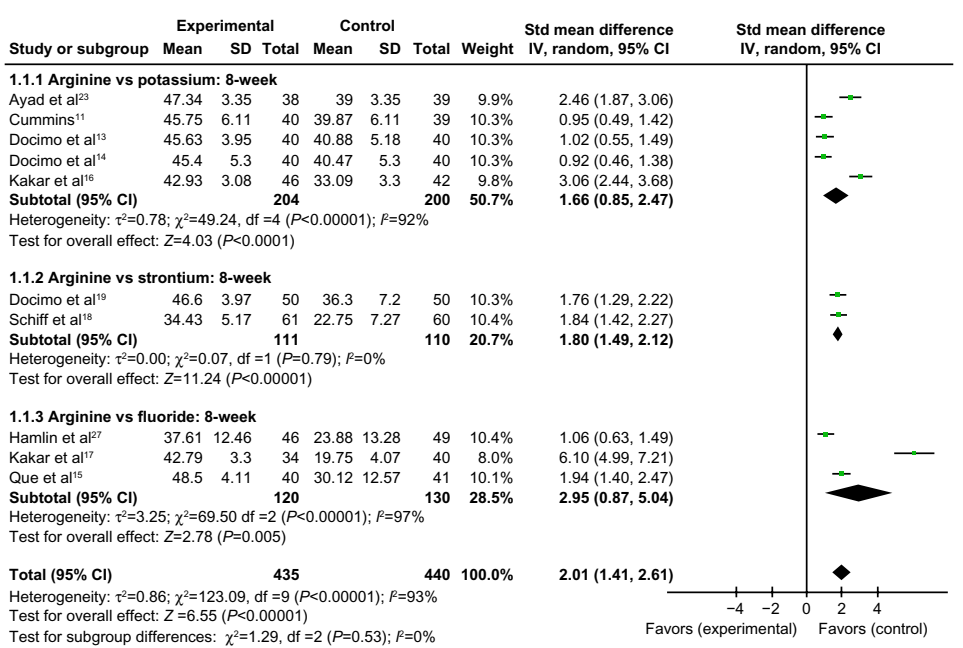

Figure I I Meta-analysis of the comparison of 8-week tactile hypersensitivity between the arginine-containing dentifrice group and control group. Abbreviations: SD, standard deviation; $\mathrm{Cl}$, confidence interval; Std, standardized; $d f$, degree of freedom.

arginine-containing dentifrice group (107 persons) and the control group (114 persons). A random-effect model was used in the meta-analysis due to statistical heterogeneity among the studies. Because the total 95\% CI lines were invalid vertical lines and the results were not significant, we concluded that the effects of the arginine-containing dentifrice and the toothpastes containing other desensitizing components did not differ significantly for the treatment of DH at $>12$ weeks (Figures 13 and 14).

\section{Sensitivity analysis}

Sensitivity analysis in which each study was eliminated individually followed by a new meta-analysis demonstrated that the direction of the effect did not change. Thus, the results of the meta-analysis were deemed reliable.

\section{Publication bias analysis}

Due to the difference in the number of included studies, we generated a funnel plot at 8 weeks. The funnel plot analysis of the 18 included studies was symmetrical, indicating no publication bias (Figure 15).

\section{Discussion}

$\mathrm{DH}$ is a clinically frequent oral health problem in which eating, breathing, and brushing teeth can cause brief, sharp pain. DH can affect the quality of daily life. Two mechanisms

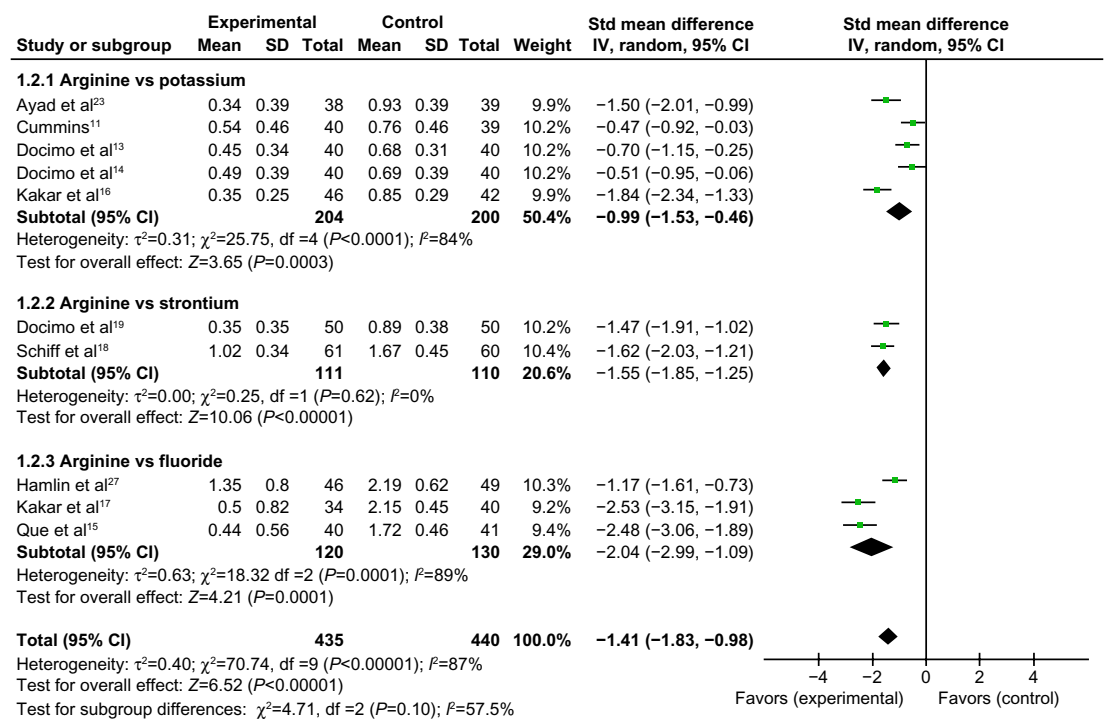

Figure 12 Meta-analysis of the comparison of 8-week air-blast hypersensitivity between the arginine-containing dentifrice group and control group. Abbreviations: SD, standard deviation; $\mathrm{Cl}$, confidence interval; Std, standardized; $d f$, degree of freedom. 


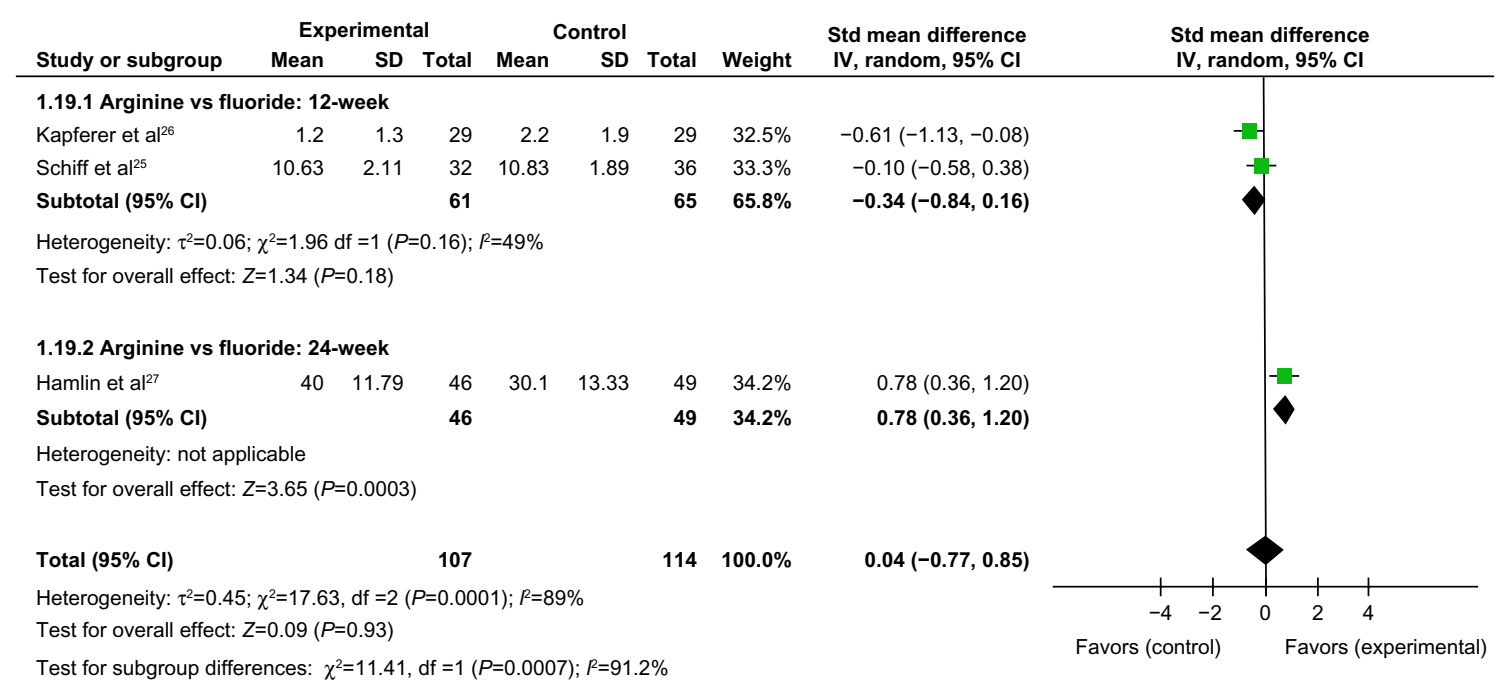

Figure 13 Meta-analysis of the comparison of > I2-week tactile hypersensitivity between the arginine-containing dentifrice group and control group. Abbreviations: SD, standard deviation; $\mathrm{Cl}$, confidence interval; Std, standardized; df, degree of freedom.

have been proposed for DH: 1) the function of odontoblasts and their processes in dentinal receptor mechanisms and 2) stimulation of pulp nerves by a hydrodynamic mechanism ${ }^{3}$ and modulation of nerve impulses in the pulp by the release of specific polypeptides during pulp injury. The most widely used and available desensitizing toothpaste ingredients are potassium compounds. In vitro studies have indicated that increases in the concentration of potassium ions in the extracellular fluid above physiological levels can induce nerve cell polarization. Thus, potassium ions may block the action potential generated in intradental nerves. Many clinical trials have demonstrated that potassium-containing toothpaste is more effective than fluoride-containing toothpaste for the treatment of $\mathrm{DH} .{ }^{34} \mathrm{~A}$ Cochrane systematic review also demonstrated that potassium-containing toothpaste is more effective for the treatment of $\mathrm{DH} .{ }^{35}$ Strontium-containing toothpastes are increasingly used to treat $\mathrm{DH}$. The tooth enamel and dentin absorb the strontium salt. Dentin has a high affinity for strontium ions. Strontium carbonate is generated when the strontium ions react with calcium carbonate in the teeth, enhancing the acid resistance of the teeth. Strontium salts can combine with other organic matter in dentin (such as oxyhemoglobin) and penetrate deeply into the tooth, significantly reducing the permeability of hard tissues and contributing to acid desensitization. Strontium chloride can calm the dentin nerve and relieve pain, slowing the conduction of the odontoblastic process and accelerating the formation of tertiary dentinogenesis. These

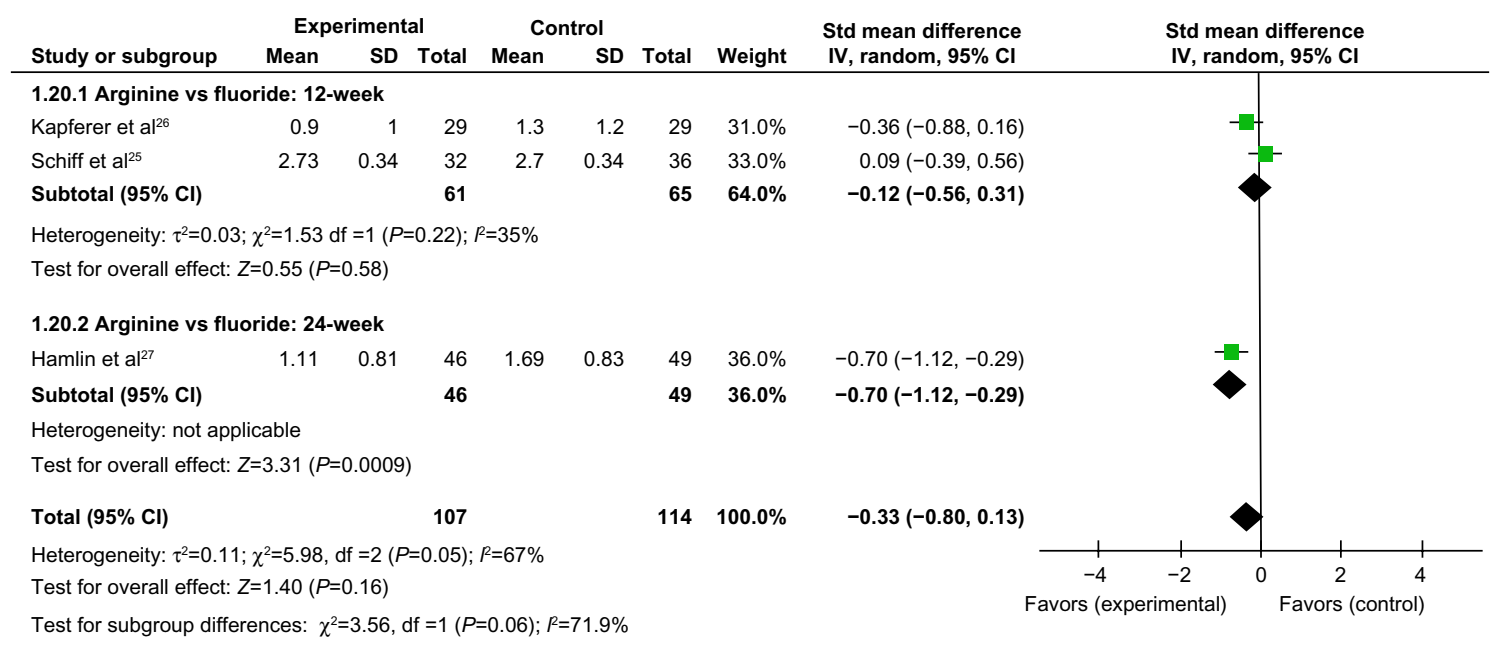

Figure 14 Meta-analysis of the comparison of $>$ I2-week air-blast hypersensitivity between the arginine-containing dentifrice group and control group. Abbreviations: SD, standard deviation; $\mathrm{Cl}$, confidence interval; Std, standardized; df, degree of freedom. 


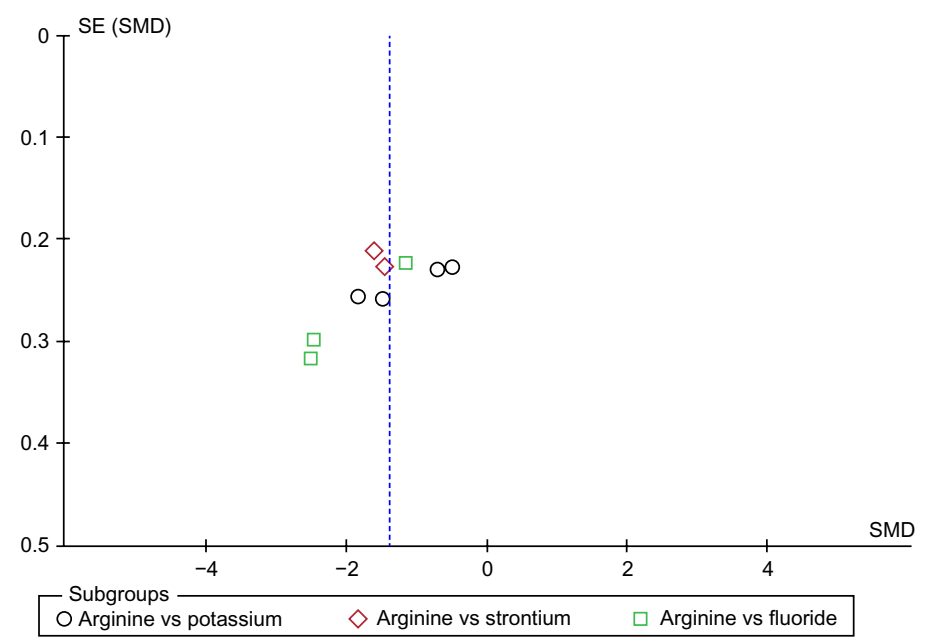

Figure 15 Funnel plot analysis of the comparison of the 8-week hypersensitivity effect between the arginine-containing dentifrice group and control group. Abbreviations: SE, standard error; SMD, standardized mean difference.

effects of strontium contribute to analgesic desensitization. A toothpaste containing $8 \%$ arginine is effective for promoting the closing of dentin tubules, and many clinical trials have demonstrated a remarkable desensitization curative effect.

\section{Superiority of the meta-analysis}

A randomized, double-blinded, controlled study is the gold standard of a curative effect evaluation. In this study, we performed a comprehensive, strictly screened, and summarized classification of RCTs of arginine-containing toothpaste for DH. We systematically evaluated the research quality of clinical studies of arginine-containing toothpaste and the treatment effect of arginine-containing toothpaste in the treatment of DH was compared to a control group in which toothpastes contain other desensitizing components. We identified evidence supporting the effective treatment of DH by argininecontaining toothpaste. The measurement of DH is subjective, and thus it is difficult to objectively measure and evaluate DH. The responses to various stimuli differ, and stimuli used to index DH must be measurable and reproducible. To avoid mutual interference, sufficient time must be allowed for dentin recovery between stimuli. Thus, studies should use at least two types of stimuli (Yeaple Electronic Pressure Sensitive Probe and Air Blast Sensitivity Assessment) to evaluate DH. This study combined the results according to the time of evaluation and the method of measuring DH. The study sample size in each group was larger than 30 subjects, which conformed to the requirement of the $\mathrm{DH}$ evaluation test and evaluation index. Data for the tactile sensitivity scores and air-blast sensitivity scores from days 0 and 3 ; weeks 2,4 , and 8 ; and more than 12 weeks were merged. We determined that the argininecontaining toothpaste was superior to the control toothpastes in relieving DH at weeks 4 and 8 , but was not significantly more effective at days 0 and 3 and week 2 as indicated by the results of the air-blast test and at more than 12 weeks as indicated by the results of the tactile sensitivity and air-blast tests. Thus, we conclude that over time, the arginine-containing toothpaste was more effective than the other toothpastes for the treatment of $\mathrm{DH}$, but beyond 12 weeks, the effects of the toothpastes were similar.

\section{Limitations of the meta-analysis}

Although the baseline of the included literature in this study was comparable, the results were for subgroup analysis. Because the data were heterogeneous, a randomization method was adopted to assign participants to study groups to ensure that the groups are balanced for known and unknown risk factors to minimize bias. For the analyses, the main causes of heterogeneity were as follows. First, there were insufficient RCTs to evaluate the effect of the arginine-containing toothpaste for DH, and evidence of systematic evaluation of its effectiveness was limited. Second, the quality of the overall research was not high. According to the evaluation standard for the quality of clinical trials (Cochrane Collaboration), the risk of bias was low. The research sites were predominantly located in the USA, Canada, and Italy, and only four research studies were performed in the People's Republic of China. Thus, data on the effectiveness of arginine-containing toothpaste for DH in Chinese populations were insufficient. Thus, regional differences in results cannot be ruled out. Third, there were individual differences in subjects, and it was difficult to treat the subjects similarly for the toothpaste dose and methods. In addition, subject compliance could not be properly evaluated. Finally, the research cycle and measurement of 
the results differed greatly. Although the clinical trials for DH treatment were normative (the evaluation results could not avoid subjective factors completely), the specific designs of the included studies differed greatly. For the same interventions, the cycle of research and the time of evaluation were different, and for the same measurement method, the result index and analysis method also differed. Thus, it was difficult to obtain a more comprehensive conclusion due to the effects of quantitatively merging the results. Because most of the studies were limited to time points of 8 weeks, longer term effects on sensitivity resistance were not observed.

\section{Conclusion}

This study examined 18 RCTs including 1,423 patients. The results support the efficacy of arginine-containing toothpaste at 4 and 8 weeks. The effects of arginine-containing toothpaste exhibited a superior therapeutic effect compared to toothpastes containing other desensitizing components as indicated by the tactile sensitivity test $(\mathrm{SMD}=1.95,95 \%$ CI $[1.14,2.76])$ and the air-blast test (SMD $=-1.60,95 \%$ CI $[-2.14,-1.05])$ at 4 weeks and the tactile sensitivity test $(\mathrm{SMD}=2.01,95 \% \mathrm{CI}[1.41,2.61])$ and the air-blast test $(\mathrm{SMD}=-1.41,95 \% \mathrm{CI}[-1.83,-0.98])$ at 8 weeks. However, no significant differences between the arginine-containing toothpaste and toothpastes containing other desensitizing components were observed at days 0 and 3 and week 2 in the air-blast test and more than 12 weeks in the tactile sensitivity and air-blast tests. However, this meta-analysis is subject to limitations (heterogeneity, quality of the evidence, limitation of time, and other factors), and stronger evidence of the superiority of arginine-containing toothpaste for $\mathrm{DH}$ for providing clinical recommendations will require additional high-quality RCTs with large sample sizes.

\section{Acknowledgments}

This work was supported by the Key Project of Medical Research Program of Chongqing Municipal Health Bureau (Grant Number: 2013-1-032) and the Science and Technology Planning Project (Grant Number: 2014-S-013) of Yubei District, Chongqing, People's Republic of China.

\section{Disclosure}

The authors report no conflicts of interest in this work.

\section{References}

1. Addy M. Dentine hypersensitivity: new perspectives on an old problem. Int Dent J. 2002;52(5):367-375.

2. Nunn JH. Prevalence and distribution of tooth wear. In: Addy M, Embery G, Edgar WM, Orchardson R, editors. Tooth Wear and Sensitivity. London, UK: Martin Dunitz; 2000:93.
3. Brännström M. Dentin sensitivity and aspiration of odontoblasts. $J A m$ Dent Assoc. 1963;66(3):366-370.

4. Kim S. Hypersensitive teeth: desensitization of pulpal sensory nerves. J Endod. 1986;12(10):482-485.

5. Blong MA, Volding B, Thrash WJ, Jones DL. Effects of a gel containing 0.4 percent stannous fluoride on dentinal hypersensitivity. Dent Hyg (Chic). 1985;59(11):489-492.

6. Snyder RA, Beck FM, Horton JE. The efficacy of a $0.4 \%$ stannous fluoride gel on root surface hypersensitivity. J Dent Res. 1985;62(3):237.

7. Thrash WJ, Dodds MW, Jones DL. The effect of stannous fluoride on dentinal hypersensitivity. Int Dent J. 1994;44(1 Suppl 1):107-118.

8. Schiff T, Saletta L, Baker RA, Winston JL, He T. Desensitizing effect of a stabilized stannous fluoride/sodium hexametaphosphate dentifrice. Compend Contin Educ Dent. 2005;26(9 Suppl 1):35-40.

9. Miller S, Truong T, Heu R, Stranick M, Bouchard D, Gaffar A. Recent advances in stannous fluoride technology: antibacterial efficacy and mechanism of action towards hypersensitivity. Int Dent J. 1994; 44(1 Suppl 1):83-98.

10. Walters PA. Dentinal hypersensitivity: a review. J Contemp Dent Pract. 2005;6(2):107-117.

11. Cummins D. Dentin hypersensitivity: from diagnosis to a break-through therapy for everyday sensitivity relief. J Clin Dent. 2009;20(1):1-9.

12. Ayad F, Ayad N, Zhang YP, DeVizio W, Cummins D, Mateo LR. Comparing the efficacy in reducing dentin hypersensitivity of a new toothpaste containing $8.0 \%$ arginine, calcium carbonate, and $1450 \mathrm{ppm}$ fluoride to a commercial sensitive toothpaste containing $2 \%$ potassium ion: an eight-week clinical study on Canadian adults. J Clin Dent. 2009; 20(1):10-16.

13. Docimo R, Montesani L, Maturo P, et al. Comparing the efficacy in reducing dentin hypersensitivity of a new toothpaste containing $8.0 \%$ arginine, calcium carbonate, and $1450 \mathrm{ppm}$ fluoride to a commercial sensitive toothpaste containing $2 \%$ potassium ion: an eight-week clinical study in Rome, Italy. J Clin Dent. 2009;20(1):17-22.

14. Docimo R, Montesani L, Maturo P, et al. Comparing the efficacy in reducing dentin hypersensitivity of new toothpaste containing $8.0 \%$ arginine, calcium carbonate, and $1450 \mathrm{ppm}$ fluoride to a benchmark commercial desensitizing toothpaste containing $2 \%$ potassium ion: an eight-week clinical study in Rome, Italy. J Clin Dent. 2009;20(4): 137-143.

15. Que K, Fu Y, Lin L, et al. Dentin hypersensitivity reduction of a new toothpaste containing $8.0 \%$ arginine, a high cleaning calcium carbonate system and $1450 \mathrm{ppm}$ fluoride: a 8-week clinical study in Chengdu, China. Am J Dent. 2010;23(Spec No A):28A-35A.

16. Kakar A, Kakar K, Sreenivasan PK, DeVizio W, Kohli R. Comparison of the clinical efficacy of a new dentifrice containing $8.0 \%$ arginine, calcium carbonate, and $1000 \mathrm{ppm}$ fluoride to a commercially available sensitive toothpaste containing $2 \%$ potassium ion on dentin hypersensitivity: a randomized clinical trial. $J$ Clin Dent. 2012;23(2):40-47.

17. Kakar A, Kakar K, Sreenivasan PK, DeVizio W, Kohli R. Comparison of the clinical efficacy in reducing dentin hypersensitivity of a new dentifrice containing $8.0 \%$ arginine, calcium carbonate, and $1000 \mathrm{ppm}$ fluoride to a commercially available sensitive toothpaste containing $2 \%$ potassium ion on dentin hypersensitivity: an eight-week clinical trial on adults in New Delhi, India. J Clin Dent. 2012;23(2):33-39.

18. Schiff T, Mateo LR, Delgado E, Cummins D, Zhang YP, DeVizio W. Clinical efficacy in reducing dentin hypersensitivity of a dentifrice containing $8.0 \%$ arginine, calcium carbonate, and $1450 \mathrm{ppm}$ fluoride compared to a dentifrice containing $8 \%$ strontium acetate and $1040 \mathrm{ppm}$ fluoride under consumer usage conditions before and after switch-over. J Clin Dent. 2011;22(4):128-138.

19. Docimo R, Perugia C, Bartolino M, et al. Comparative evaluation of the efficacy of three commercially available toothpastes on dentin hypersensitivity reduction: an eight-week clinical study. Am J Clin Dent. 2011;22(4):121-127.

20. Li Y, Lee S, Zhang YP, Delagado E, Devizio W, Matco LR. Comparison of clinical efficacy of three toothpastes in reducing dentin hypersensitivity. J Clin Dent. 2011;22(4):113-120. 
21. Nathoo S, Delgado E, Zhang YP, DeVizio W, Cummins D, Mateo LR. Comparing the efficacy in providing instant relief of dentin hypersensitivity of a new toothpaste containing $8.0 \%$ arginine, calcium carbonate, and $1450 \mathrm{ppm}$ fluoride relative to a benchmark desensitizing toothpaste containing $2 \%$ potassium ion and $1450 \mathrm{ppm}$ fluoride, and to a control toothpaste with $1450 \mathrm{ppm}$ fluoride: a three-day clinical study in New Jersey, USA. J Clin Dent. 2009;20(4):123-130.

22. Fu Y, Li X, Que K, et al. Instant dentin hypersensitivity relief of a new desensitizing dentifrice containing $8.0 \%$ arginine, a high cleaning calcium carbonate system and 1450 ppm fluoride: a 3-day clinical study in Chengdu, China. Am J Dent. 2010;23(Spec No A):20A-27A.

23. Ayad F, Ayad N, Delgado E, et al. Comparing the efficacy in providing instant relief of dentin hypersensitivity of a new toothpaste containing $8.0 \%$ arginine, calcium carbonate, and $1450 \mathrm{ppm}$ fluoride to a benchmark desensitizing toothpaste containing $2 \%$ potassium ion and $1450 \mathrm{ppm}$ fluoride, and to a control toothpaste with $1450 \mathrm{ppm}$ fluoride: a three-day clinical study in Mississauga, Canada. J Clin Dent. 2009;20(4):115-122.

24. Hamlin D, Williams KP, Delgado E, Zhang YP, DeVizio W, Mateo LR. Clinical evaluation of the efficacy of a desensitizing paste containing $8 \%$ arginine and calcium carbonate for the in-office relief of dentin hypersensitivity associated with dental prophylaxis. Am J Dent. 2009; 22(Spec No A):16A-20A.

25. Schiff T, Delgado E, Zhang YP, Cummins D, DeVizio W, Mateo LR. Clinical evaluation of the efficacy of an in-office desensitizing paste containing $8 \%$ arginine and calcium carbonate in providing instant and lasting relief of dentin hypersensitivity. Am J Dent. 2009;22(Spec No A): $8 \mathrm{~A}-15 \mathrm{~A}$.

26. Kapferer I, Pflug C, Kisielewsky I, Giesinger J, Beier US, Dumfahrt H. Instant dentin hypersensitivity relief of a single topical application of an in-office desensitizing paste containing $8 \%$ arginine and calcium carbonate: a split-mouth, randomized-controlled study. Acta Odontol Scand. 2013;71(3-4):994-999.
27. Hamlin D, Mateo LR, Dibart S, Delgado E, Zhang YP, DeVizio W. Comparative efficacy of two treatment regimens combining in-office and at-home program for dentin hypersensitivity relief: a 24-week clinical study. Am J Dent. 2012;25(3):146-152.

28. He T, Chang J, Cheng R, Li X, Sun L, Biesbrock AR. Clinical evaluation of the fast onset and sustained sensitivity relief of a $0.454 \%$ stannous fluoride dentifrice compared to an $8.0 \%$ arginine-calcium carbonate-sodium monofluorophosphate dentifrice. Am J Dent. 2011; 24(6):336-340.

29. He T, Cheng R, Biesbrock AR, Chang A, Sun L. Rapid desensitizing efficacy of a stannous-containing sodium fluoride dentifrice. J Clin Dent. 2011;22:40-45.

30. Higgins JPT, Green S, editors. Cochrane Handbook for Systematic Reviews of Interventions. Version 5.1.0. London, UK: Cochrane; 2011. Available from: http://www.cochrane-handbook.org. Accessed November 25, 2015.

31. Atkins D, Best D, Briss PA, et al; GRADE Working Group. Grading quality of evidence and strength of recommendations. BMJ. 2004; 328(7454):1490.

32. Guyatt G, Oxman AD, Akl EA, et al. GRADE guidelines: 1. Introduction-GRADE evidence profiles and summary of findings tables. J Clin Epidemiol. 2011;64(4):383-394.

33. Guyatt GH, Oxman AD, Vist GE, et al. GRADE: an emerging consensus on rating quality of evidence and strength of recommendations. BMJ. 2008;336(7650):924-926.

34. Orchardson R, Gillam DG. The efficacy of potassium salts as agents for treating dentin hypersensitivity. J Orofac Pain. 2000;14(1):9-19.

35. Poulsen S, Errboe M, Lescay Mevil Y, Glenny AM. Potassium containing toothpastes for dentine hypersensitivity. Cochrane Database Syst Rev. 2006;19(3):CD001476.
Clinical, Cosmetic and Investigational Dentistry

\section{Publish your work in this journal}

Clinical, Cosmetic and Investigational Dentistry is an international, peer-reviewed, open access, online journal focusing on the latest clinical and experimental research in dentistry with specific emphasis on cosmetic interventions. Innovative developments in dental materials, techniques and devices that improve outcomes and patient satisfac-

\section{Dovepress}

tion and preference will be highlighted. The manuscript management system is completely online and includes a very quick and fair peerreview system, which is all easy to use. Visit http://www.dovepress. com/testimonials.php to read real quotes from published authors. 